\title{
mesure des contraintes au sein d'un massif analogique de Schneebeli
}

\author{
par \\ J.-C. Faugeras \\ Assistant au C.U.S.T. de Clermont-Ferrand \\ R. Gourves \\ Maître-Assistant au C.U.S.T. de Clermont-Ferrand \\ Groupe de Recherche Génie Civil de I'Université de Clermont II
}

\section{Introduction}

La connaissance de l'état de contrainte dans les milieux pulvérulents pose encore à l'heure actuelle de sérieux problèmes que de nombreux chercheurs ont plusieurs fois mis en évidence.

Schneebeli (1) a montré la possibilité de réaliser un milieu pulvérulent obéissant à la loi de Coulomb, par un empilage de petits cylindres parallèles, horizontaux et de même longueur. Un tel modèle permet l'étude de problèmes à deux dimensions et de nombreux auteurs $(2,3,4,5)$ l'ont utilisé notamment pour mieux comprendre les schémas de rupture du sol de fondation, l'équilibre limite des coins de poussée et de butée, la stabilité des murs de terre armée, etc... Jusqu'à présent ce modèle ne permettait que de visualiser les différents phénomènes bien que certaines tentatives aient été faites $(6,7)$ pour déterminer les contraintes dans ce type de milieu sans donner de résultats satisfaisants, la meilleure méthode consistant cependant à utiliser des capteurs à friction (8). La méthode que nous nous proposons de présenter ici repose sur un principe simple et permet d'obtenir l'état de contrainte en tous points du milieu. Si quelques problèmes de précision dans les résultats obtenus semblent continuer à se poser, l'amélioration du processus expérimental nous conduira dans un proche avenir à définir de façon très précise le champ de contrainte.

\section{Principe de la méthode}

Dans un massif constitué par un empilement de cylindres, la force nécessaire à déplacer l'un d'entre eux selon son axe longitudinal est fonction de la profondeur à laquelle il se situe, le milieu étant pesant. La méthode mise au point est fondée sur cette constatation.

\subsection{Méthode pour un seul cylindre}

On peut représenter les forces agissant sur un seul cylindre comme indiqué à la figure $1 \mathrm{a}$. Nous ne prenons en compte que les forces radiales, la résistance à l'enfoncement des rouleaux n'étant proportionnelle qu'à ces dernières dans la direction considérée. Nous pouvons écrire :

$F_{T}=\operatorname{tg} \delta \cdot \Sigma F_{i}$

avec : $F_{\mathrm{T}}$ force nécessaire à déplacer un rouleau selon son axe longitudinal

$\delta$ angle de frottement longitudinal des rouleaux $\Sigma F_{1}$ somme des forces appliquées.

Sous l'action des $F_{i}$ le rouleau est en équilibre. Nous pouvons donc définir la pression isotrope équivalente à laquelle est soumis le rouleau par l'expression :

$\Sigma F_{i}=p \times 2 \pi r$ pour une longueur unité, $r$ étant le rayon du cylindre.

Dans la mesure où nous sommes en présence d'un milieu granulaire, il est nécessaire d'introduire la notion de contrainte macroscopique qui ne peut être utilisée qu'en considérant un nombre important de rouleaux. De plus à l'intérieur du massif analogique se créent des voûtes de charge, ce qui implique que certains grains ne sont soumis qu'à la seule force de pesanteur.

Cette méthode ne peut donc présenter un intérêt qu'au . niveau d'une étude statistique du comportement de grains pris singulièrement.

\subsection{Méthode pour plusieurs cylindres}

Dans ce cas nous déplaçons simultanément plusieurs rouleaux, toujours selon une distance parallèle à leur axe longitudinal et nous appelons « découpe $*$ la limite de l'empreinte créée dans le massif par ce déplacement. Nous nous intéressons au cas où cette découpe est quasi circulaire ou à ellipse d'inertie circulaire (figures $1 \mathrm{~b}$ et $1 \mathrm{c}$ ).

En gardant les mêmes notations que précédemment, nous pouvons écrire:

$\mathrm{F}_{\mathrm{T}}=\operatorname{tg} \delta \cdot \Sigma \mathrm{F}_{\mathrm{i}}$ 


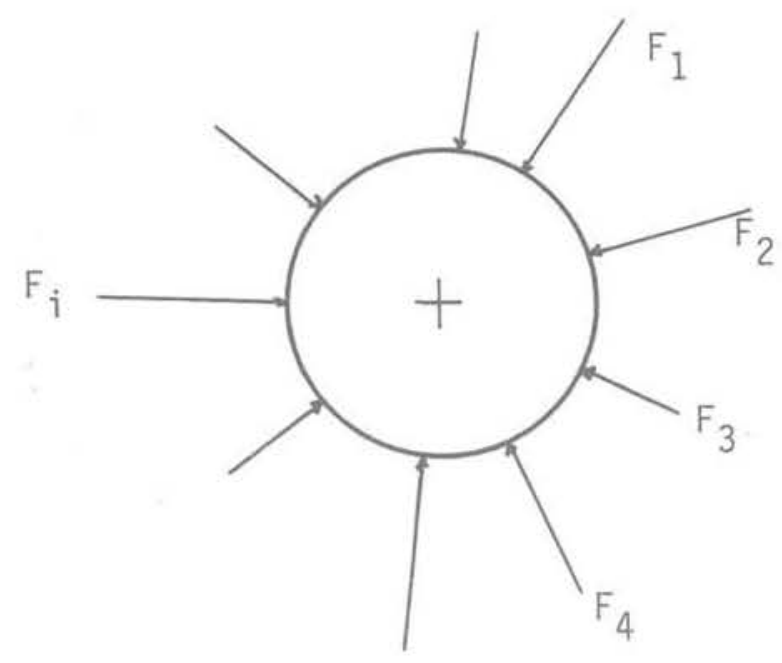

(a)

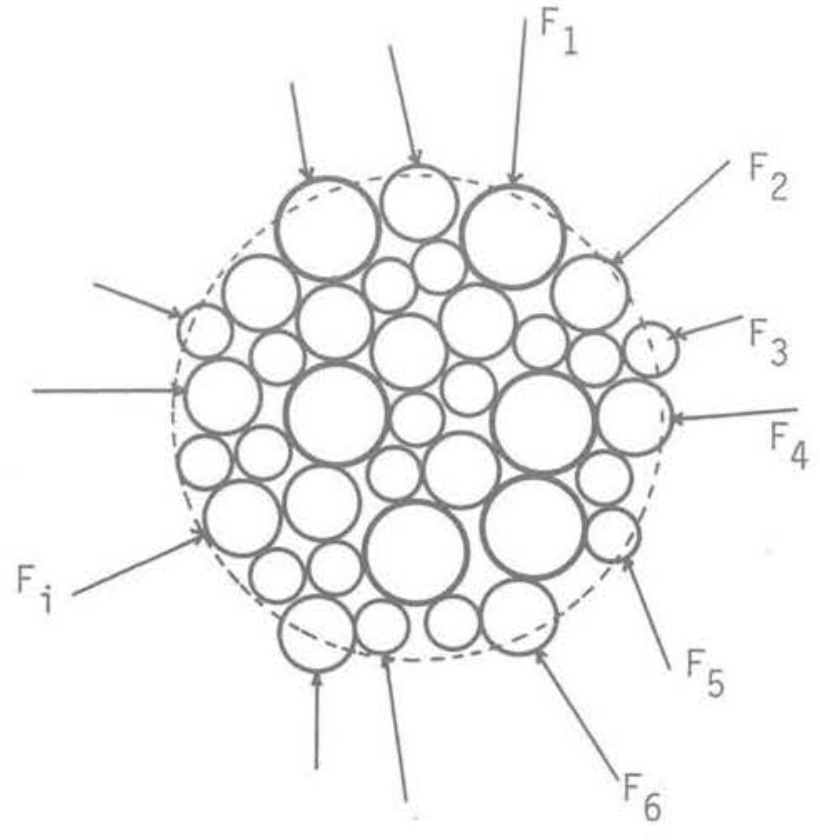

(b)

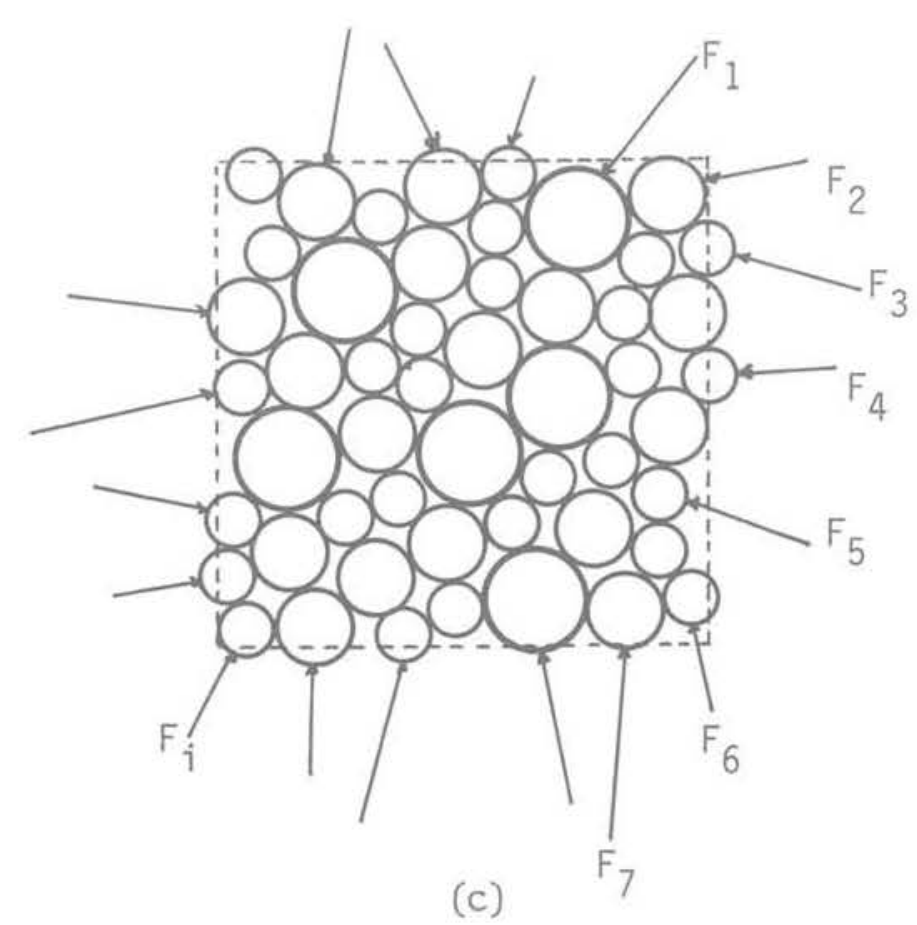

Fig. 1 Schéma des forces agissant sur les rouleaux

et de même :

$\Sigma F_{1}=p \times 2 \pi R \quad$ (R rayon de la découpe).

La figure 2 montre l'évolution de la pression $p$ en fonction de la profondeur au sein d'un massif non chargé. Nous obtenons une bonne corrélation entre ces deux paramètres, mais cette méthode, bien que satisfaisante, ne peut cependant pas être exploitée pour la détermination du champ de contrainte.

\subsection{Méthode conduisant à la détermination du champ de contrainte}

Au vu des résultats précédents nous avons pensé qu'il était possible d'obtenir l'état de contrainte en utilisant un autre type de découpe, comme le montre la figure 3.

Dans un milieu continu, la notion de contrainte est définie comme étant la limite du rapport dF/dS lorsque dS tend vers zéro. Si nous considérons une surface contenue dans un plan coupant un matériau granulaire, nous appellerons contrainte normale la somme des composantes normales des forces s'exerçant sur les particules, divisée par l'aire de cette surface. Dans notre cas, $\sigma_{z}$ est donc la contrainte moyenne macroscopique qui s'exerce sur une facette de normale $\mathrm{Oz}$. II est de même possible de définir une contrainte normale $\sigma_{\mathrm{x}}$.

L'expression de la force nécessaire pour déplacer les rouleaux selon leur axe longitudinal peut être mise sous la forme:

$F_{z}=\lambda \sigma_{z}+\mu \sigma_{x}$

$\lambda$ et $\mu$ sont des coefficients qui dépendent des caractéristiques géométriques de la plaque utilisée pour déplacer les rouleaux. 


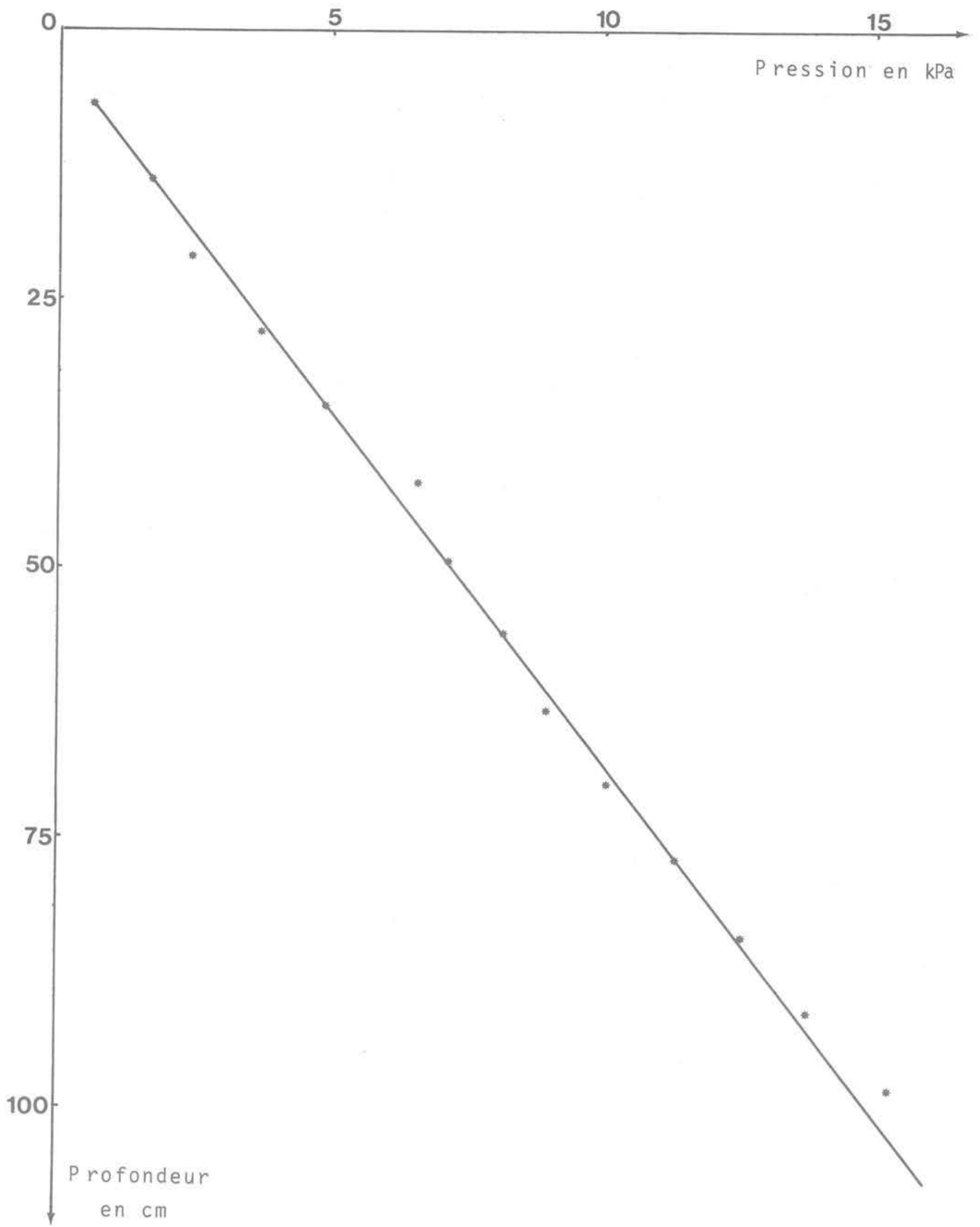

Fig. 2 Variation de pression isotrope au sein du massif . 


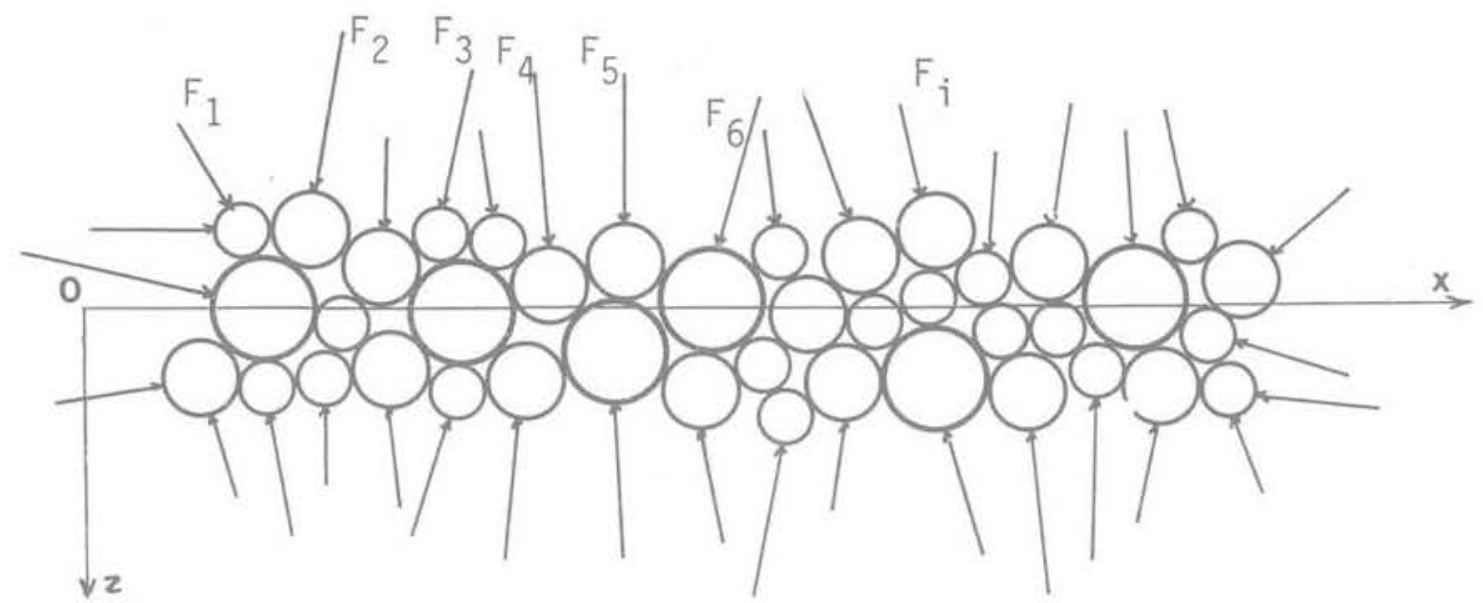

Fia. 3 Découpe obtenue avec une plaque

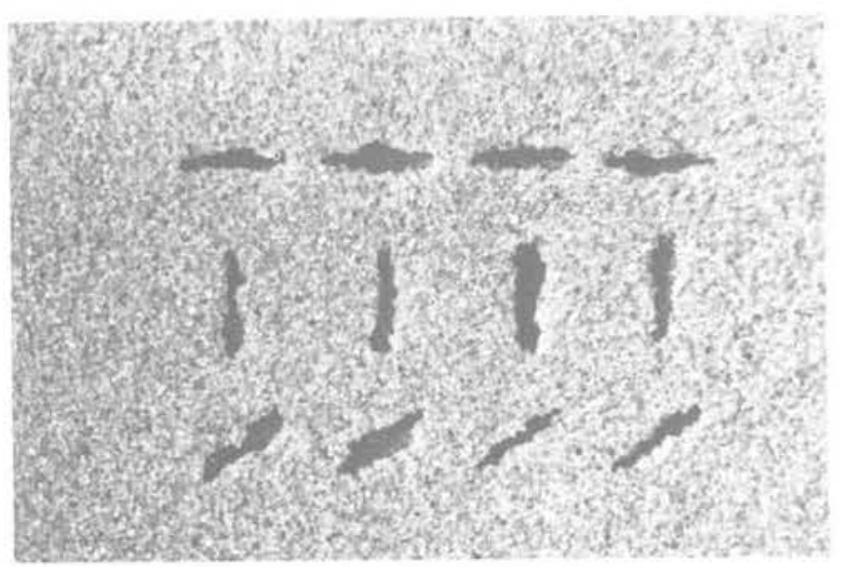

Fig. 4 Formes des découpes

Fig. 5 Schéma de principe de l'appareil de compression biaxial

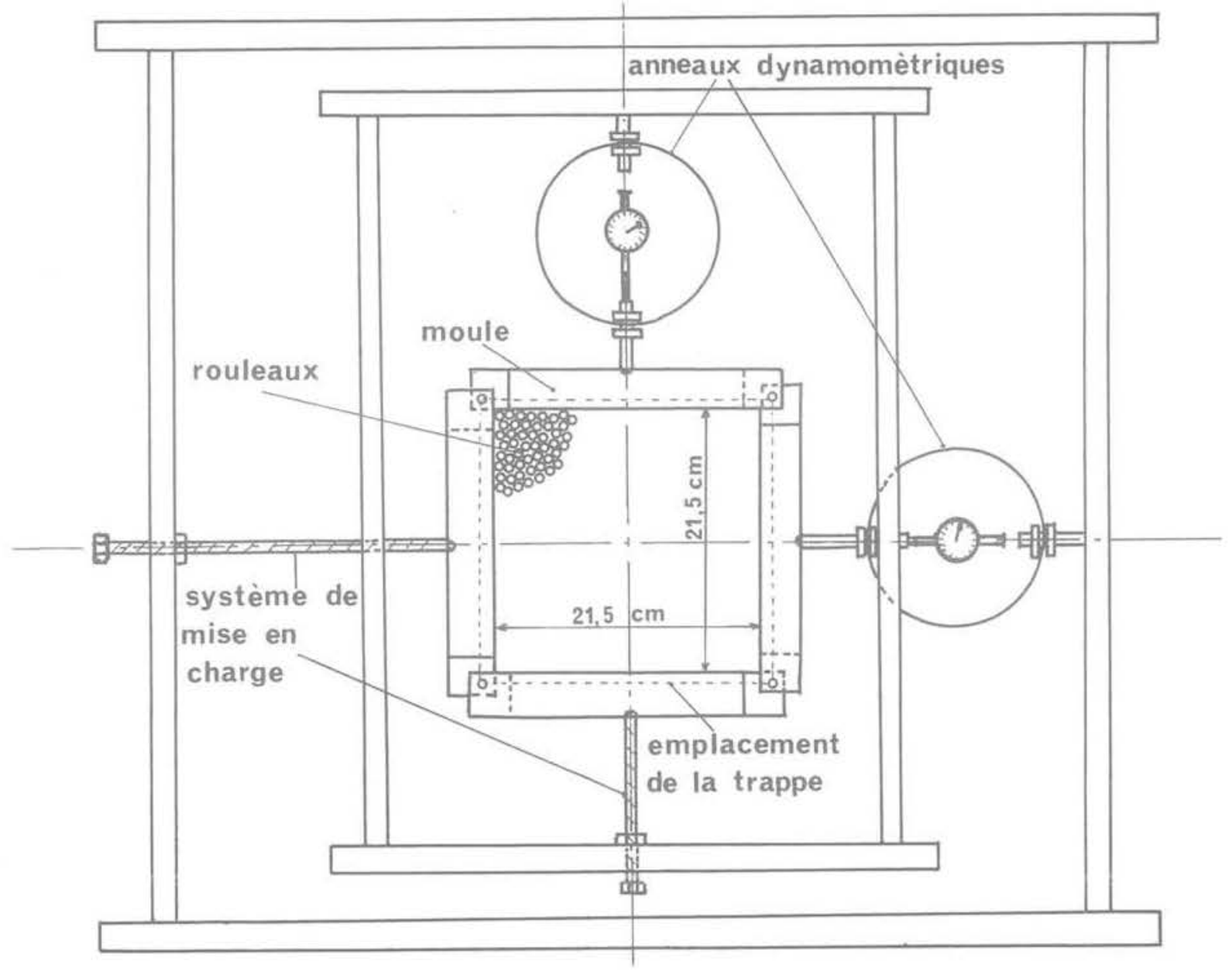


De plus, quelle que soit l'orientation de la découpe dans le plan $x O z$, le contour de celle-ci présente la même configuration (fig. 4). L'isotropie nous permet donc d'écrire :

$F_{\theta}=\lambda \sigma_{\theta}+\mu \sigma_{\theta+\pi / 2}$

avec : $\theta$ angle que fait le grand axe de la découpe avec la direction $\mathrm{Ox}$.

\section{Détermination des coefficients $\lambda$ et $\mu$}

Pour déterminer les coefficients $\lambda$ et $\mu$, il faut opérer dans un champ de contrainte connu. Pour ce faire nous avons conçu une machine de compression biaxiale.

\subsection{Appareillage utilisé}

Nous avons choisi d'utiliser des rouleaux en PVC en raison de leur faible poids volumique $\left(13,4 \mathrm{kN} / \mathrm{m}^{3}\right)$. Leurs diamètres sont de 2,3 et $4 \mathrm{~mm}$ également répartis en poids et leur longueur de $6 \mathrm{~cm}$. Leur angle de frottement $\varphi$ a été déterminé de deux façons différentes :

- à l'appareil biaxial : $20^{\circ}<\varphi<32^{\circ}$;

- la mesure de la pente de talus naturel a donné des résultats très légèrement inférieurs : $27^{\circ}<\varphi<31^{\circ}$.

Pour mesurer les $F_{\theta}$ nous utilisons un dynamomètre de 10 daN de capacité dont la précision est de $\pm 0,5 \%$ de la valeur de fin d'échelle. En bout, une plaque de $5 \mathrm{~cm}$ de large et de $1 \mathrm{~mm}$ d'épaisseur permet de pousser sur les rouleaux. Comme le montre le schéma de la figure 5, la machine de compression biaxiale est constituée par un moule en quatre éléments mobiles les uns par rapport aux autres et contenant les rouleaux. Les parois en contact avec les rouleaux sont recouvertes d'un lubrifiant à sec pour éviter la création de voûtes de charge. Deux cadres métalliques indépendants assurent la mise en charge par un système vis-écrou. Les efforts appliqués sont mesurés par deux anneaux dynamomètriques, ce qui permet de déterminer l'état de contrainte.

L'ensemble est posé sur une plaque à surface lisse, des rouleaux étant entreposés entre les différents éléments constituants l'appareil et la plaque, ceci afin de minimiser les frottements. Une trappe placée sous le moule permet de pousser sur les rouleaux en vue de la mesure des $F_{\theta}$.

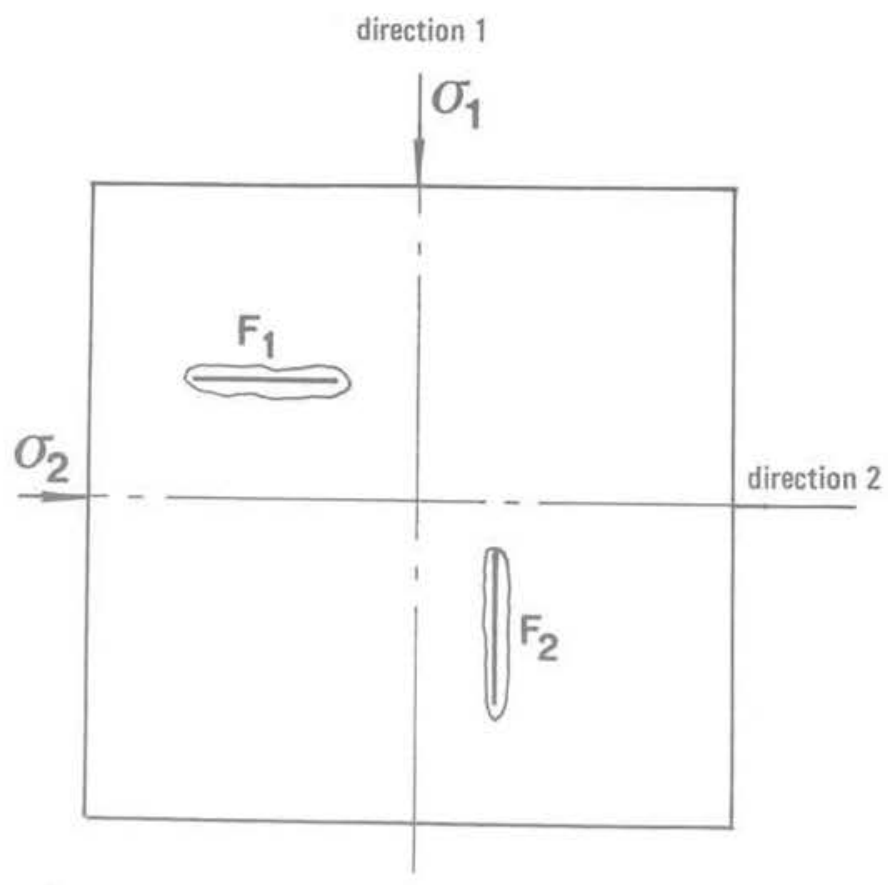

Fig. 6 Notations utilisées

\subsection{Mise en œuvre des essais et résultats}

Nous adoptons les notations de la figure 6. Après plusieurs tentatives, nous avons adopté la méthode suivante pour la détermination de $\lambda$ et $\mu$;

- application d'un champ de contrainte constant $\sigma_{1}$, $\sigma_{2}$;

- mesure des $F_{1}$ en 15 points différents (fig. $7 \mathrm{a}$ );

- déchargement du système et remise en place des rouleaux, ceci pour éviter la création de lignes préférentielles de glissement;

- application du même champ de contrainte que précédemment;

- mesure des $F_{1}$ à nouveau en 15 points mais différents des précédents (fig. 7 b);

- déchargement du système et remise en place des rouleaux.
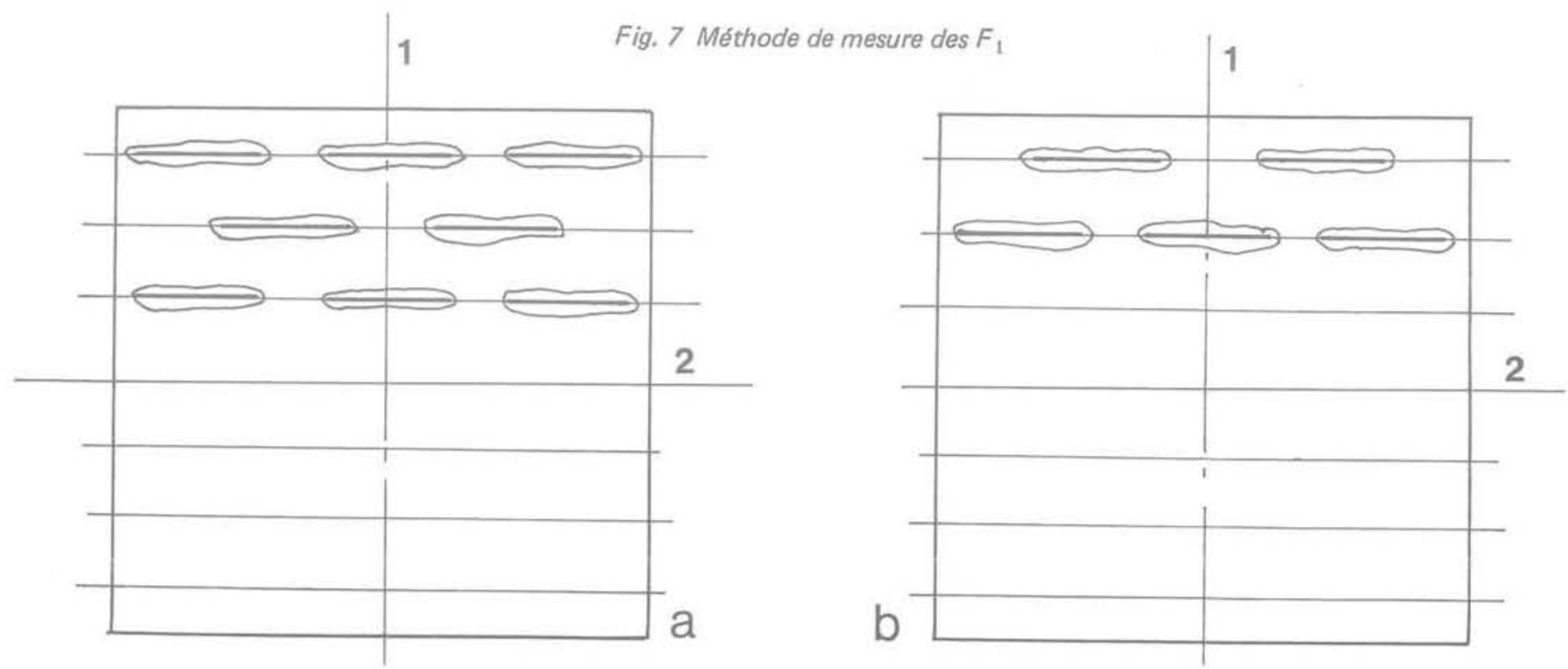


$$
\sigma_{1}=61,2 \mathrm{kPa} \quad \sigma_{2}=31,1 \mathrm{kPa} \quad \sigma_{1} / \sigma_{2}=1,97
$$

Valeurs des $F_{1}$

\begin{tabular}{|l|l|l|l|l|l|c|}
\cline { 5 - 6 } & \multicolumn{2}{c|}{$\begin{array}{c}\text { Moyenne par } \\
\text { ligne }\end{array}$} \\
\hline ligne 1 & 7,06 & 7,05 & 7,80 & 6,80 & 6,50 & 7,04 \\
ligne 2 & 6,80 & 6,80 & 6,60 & 7,15 & 7,20 & 6,91 \\
ligne 3 & 6,70 & 8,05 & 7,75 & 7,35 & 6,90 & 7,35 \\
ligne 4 & 7,00 & 6,70 & 7,70 & 7,05 & 6,75 & 7,04 \\
ligne 5 & 6,30 & 7,20 & 7,30 & 7,50 & 7,30 & 7,12 \\
ligne 6 & 7,75 & 5,85 & 7,70 & 7,80 & 7,35 & 7,29 \\
\hline moyenne & 6,93 & 6,94 & 7,47 & 7,27 & 7,00 & 7,12 \\
\hline
\end{tabular}

Valeur des $F_{2}$

\begin{tabular}{|l|l|l|l|l|l|l|}
\cline { 5 - 6 } & \multicolumn{2}{c|}{$\begin{array}{c}\text { Moyenne par } \\
\text { ligne }\end{array}$} \\
\hline ligne 1 & 5,10 & 5,10 & 4,65 & 3,90 & 4,50 & 4,65 \\
ligne 2 & 5,25 & 5,00 & 4,25 & 4,10 & 5,45 & 4,81 \\
ligne 3 & 5,45 & 5,55 & 4,55 & 5,30 & 5,35 & 5,24 \\
ligne 4 & 4,20 & 4,85 & 4,85 & 5,15 & 5,05 & 4,82 \\
ligne 5 & 4,70 & 5,35 & 5,05 & 5,05 & 5,45 & 5,12 \\
ligne 6 & 4,05 & 4,45 & 4,35 & 4,40 & 6,15 & 4,68 \\
\hline moyenne & 4,79 & 5,05 & 4,62 & 4,65 & 5,32 & 4,89 \\
\hline
\end{tabular}

$\lambda_{\text {moyen }}=10,22 \mathrm{~cm}^{2} \quad \mu_{\text {moyen }}=2,80 \mathrm{~cm}^{2}$

Fig. 8 Exemple de résultats obtenus au biaxial

Pour un même déviateur nous obtenons ainsi 30 mesures de $F_{1}$. La mesure des $F_{2}$ s'effectue selon le même principe. Un exemple des résultats ainsi obtenus est donné figure 8.

La manipulation décrite ci-dessus est effectuée cinq fois. Le déviateur est alors fixé à une nouvelle valeur et le processus est recommencé.

Connaissant $F_{1}, F_{2}, \sigma_{1}$ et $\sigma_{2}$ il est alors possible de déterminer $\lambda$ et $\mu$ par les relations :

$\lambda=\frac{\sigma_{1} F_{1}-\sigma_{2} F_{2}}{\sigma_{1}^{2}-\sigma_{2}^{2}}$ et $\mu=\frac{\sigma_{1} F_{2}-\sigma_{2} F_{1}}{\sigma_{1}^{2}-\sigma_{2}^{2}}$.

Les résultats que nous présentons ci-après sont des valeurs moyennes:

\begin{tabular}{c|c|c|c|c|c|c}
\hline$\sigma_{1} / \sigma_{2}$ & $\begin{array}{c}\text { moyenne } F_{1} \\
\text { en daN }\end{array}$ & $\begin{array}{c}\text { écart-type } \\
F_{1}\end{array}$ & $\begin{array}{c}\text { moyenne } F_{2} \\
\text { en daN }\end{array}$ & $\begin{array}{c}\text { écart-type } \\
F_{2}\end{array}$ & $\begin{array}{c}\lambda \\
\text { en } \mathrm{cm}^{2} \text { en } \mathrm{cm}^{2}\end{array}$ \\
\hline 1,97 & 6,99 & 0,55 & 4,88 & 0,59 & 10,02 & 2,86 \\
1,46 & 5,70 & 0,56 & 4,41 & 0,58 & 11,04 & 2,08 \\
1,18 & 5,77 & 0,57 & 5,21 & 0,54 & 10,47 & 2,47 \\
\hline
\end{tabular}

D'après ces résultats, nous pouvons considérer $\lambda$ et $\mu$ comme constants et indépendants du déviateur. Dans la suite de cette étude, nous prendrons les valeurs moyennes suivantes:

$\lambda=10,5 \mathrm{~cm}^{2} \quad \mu=2,6 \mathrm{~cm}^{2}$.

\section{Traitement automatique des données}

Les résultats que nous présentons ultérieurement ont été obtenus sur un massif de 1,20 m de hauteur et de 2,10 m de longueur. La constitution de ce massif était bien évidemment identique à celle de l'échantillon testé à l'appareil de compression biaxial.

Pour faciliter l'analyse des résultats, nous avons effectué un maillage du massif de $0,07 \times 0,07 \mathrm{~m}$, ce qui permet de prendre 392 mesures. Compte tenu de ce nombre relativement important, nous avons utilisé pour l'exploitation des résultats un moyen de calcul automatique.

Les différentes valeurs des mesures sont stockées sous forme de tableaux de 14 lignes et 28 colonnes correspondant au maillage du massif (fig. 9). II faut noter que les résultats obtenus par la méthode de mesure utilisée présentent une assez grande dispersion. Pour diminuer ces écarts deux solutions peuvent être envisagées. Nous pouvons soit effectuer un grand nombre de mesures en chaque point du massif, soit effectuer un lissage des valeurs obtenues. Pour des raisons de mise en œuvre, il était préférable d'utiliser la deuxième solution. Comme le montrent les courbes de la figure 10 , nous constatons une nette amélioration. Après plusieurs essais, nous avons opté pour le schéma de lissage suivant:

\begin{tabular}{|c|c|}
\hline $\mathrm{A}_{i-1, j-1}$ & $\begin{array}{c}A_{i-1,1} \\
(2)\end{array}$ \\
\hline $\begin{array}{l}A_{1,1-1} \\
\text { (2) }\end{array}$ & $\begin{array}{l}A_{i, i} \\
(3)\end{array}$ \\
\hline$A_{i+1, j-1}$ & $\begin{array}{c}A_{i+1.1} \\
\text { (2) }\end{array}$ \\
\hline
\end{tabular}

La valeur lissée du terme $A_{i, j}$ est telle que :

$A_{i, j}=\left[A_{i, 1} \times 3+\left(A_{i-1, j}+A_{i+1, j}+A_{i, j-1}+A_{i, j+1}\right) \times 2\right.$

$$
\left.+\left(A_{i-1, j-1}+A_{i+1, i+1}+A_{i-1, j+1}+A_{i+1, j-1}\right)\right] / 15 \text {. }
$$

La suite des calculs s'effectue à partir de ces valeurs lissées :

- détermination de $\sigma_{x}$ et $\sigma_{z}$ :

nous avons: $F_{z}=\lambda \sigma_{z}+\mu \sigma_{x}$ et $F_{x}=\lambda \sigma_{x}+\mu \sigma_{z}$ nous en déduisons :

$\sigma_{z}=\frac{1}{\lambda^{2}-\mu^{2}}\left(\lambda F_{z}-\mu F_{X}\right)$

$\sigma_{x}=\frac{1}{\lambda^{2}-\mu^{2}}\left(\lambda F_{x}-\mu F_{z}\right) ;$

- détermination de $\theta$ :

appelons $\theta$ l'angle dont il faut faire tourner le repère initial pour obtenir le repère des contraintes principales. Un calcul simple à partir du cercle de Mohr des contraintes, en exprimant les contraintes en fonction des forces $\mathrm{F}$ définies précédemment conduit à :

$\operatorname{tg} 2 \theta=\frac{F_{z}+F_{X}-2 F_{45}}{F_{z}-F_{X}}$

$F_{45}$ étant la force mesurée pour une découpe à $45^{\circ}$. On voit ainsi que $\theta$ est indépendant de $\lambda$ et $\mu$ et qu'il suffit de trois manipulations pour le déterminer, la méthode étant classique en extensométrie :

- détermination de $\sigma_{1}$ et $\sigma_{2}$.

Les contraintes principales sont déterminées par les expressions connues :

$\sigma_{1}=\frac{\sigma_{z}+\sigma_{x}}{2}+\frac{\sigma_{z}-\sigma_{x}}{2 \cos 2 \theta}$ et $\sigma_{2}=\frac{\sigma_{z}+\sigma_{x}}{2}-\frac{\sigma_{z}-\sigma_{x}}{2 \cos 2 \theta}$ 


\begin{tabular}{|c|c|c|c|c|c|c|c|c|c|c|c|c|c|c|}
\hline & 1 & 2 & 3 & 4 & 5 & 6 & 7 & 8 & 9 & 10 & 11 & 12 & 13 & 14 \\
\hline 1 & $* * * * * *$ & $* * * * * *$ & ******** & $* * * * * * *$ & $* * * * * * *$ & ******* & $* * * * * *$ & $* * * * * *$ & $* * * * * *$ & $* * * * * *$ & ******* & $* * * * * *$ & $* * * * * *$ & $* * * * * *$ \\
\hline 2 & $* * * * * *$ & 0.15 & 0.15 & 0.10 & 0.10 & $* * * * * *$ & $* * * * * *$ & 0.05 & 0.05 & $* * * * * *$ & 0.05 & 0.05 & 0.05 & 0.05 \\
\hline 3 & 0.20 & 0.25 & 0.20 & 0.20 & 0.30 & 0.25 & 0.25 & 0.45 & 0.15 & 0.20 & 0.20 & 0.20 & 0.20 & 0.20 \\
\hline 4 & 0.40 & 0.30 & 0.40 & 0.40 & 0.30 & 0.30 & 0.45 & 0.40 & 0.40 & 0.35 & 0.40 & 0.35 & 0.45 & 0.30 \\
\hline 5 & 0.60 & 0.55 & 0.50 & 0.50 & 0.55 & 0.50 & 0.45 & 0.70 & 0.50 & 0.45 & 0.50 & 0.35 & 0.55 & 0.55 \\
\hline 6 & 0.70 & 0.65 & 0.65 & 0.70 & 0.60 & 0.65 & 0.55 & 0.60 & 0.55 & 0.60 & 0.65 & 0.55 & 0.55 & 0.65 \\
\hline 7 & 0.70 & 0.45 & 0.65 & 0.70 & 0.70 & 0.80 & 0.60 & 0.80 & 0.60 & 0.65 & 0.65 & 0.75 & 1.00 & 0.85 \\
\hline 8 & 0.75 & 0.80 & 0.80 & 0.80 & 0.80 & 0.85 & 0.90 & 1.05 & 0.80 & 0.85 & 1.15 & 1.00 & 1.05 & 0.75 \\
\hline 9 & 0.95 & 1.10 & 0.90 & 0.70 & 0.95 & 1.10 & 1.05 & 0.90 & 0.95 & 0.85 & 1.00 & 1.05 & 1.25 & 1.15 \\
\hline 10 & 1.05 & 1.20 & 1.10 & 1.05 & 1.00 & 1.20 & 1.15 & 1.05 & 2.00 & 0.95 & 1.15 & 1.15 & 1.50 & 1.40 \\
\hline 11 & 1.20 & 1.45 & 1.10 & 1.35 & 1.25 & 1.30 & 1.15. & 1.25 & 1.25 & 1.05 & 1.05 & 1.50 & 1.55 & 1.45 \\
\hline 12 & 1.10 & 1.25 & 1.40 & 1.55 & 1.40 & 1.35 & 1.40 & 1.15 & 1.30 & 1.30 & 1.20 & 1.60 & 1,55 & 1.70 \\
\hline 13 & 1.70 & 1.60 & 1.75 & 1.45 & 1.50 & 1.55 & 1.60 & 1.85 & 1.50 & 1.25 & 1.55 & 1.75 & 1.65 & 2.30 \\
\hline 14. & 1.85 & 1,75 & 2.25 & 2.25 & 1.65 & 1.85 & 2.25 & 2.25 & 1.70 & 1.70 & 1.65 & 2.00 & 2.15 & 2.10 \\
\hline & 15 & 16 & 17 & 18 & 19 & 20 & 21 & 22 & 23 & 24 & 25 & 26 & 27 & 28 \\
\hline 1 & $* * * * * *$ & $* * * * * *$ & $* * * * * *$ & $* * * * * * *$ & ******* & $* * * * * *$ & ******* & $* * * * * *$ & $* * * * * *$ & $* * * * * *$ & $* * * * * *$ & $* * * * * *$ & $* * * * * *$ & $* * * * * *$ \\
\hline 2 & 0.05 & 0.05 & $* * * * * *$ & 0.05 & 0.05 & 0.05 & 0.05 & 0.05 & 0,10 & 0.10 & 0.05 & 0,10 & 0.10 & 0.10 \\
\hline 3 & 0.20 & 0.15 & 0.20 & 0.25 & 0.25 & 0.20 & 0.15 & 0.20 & 0.25 & 0.25 & 0.25 & 0.20 & 0.20 & 0.25 \\
\hline 4 & 0.30 & 0.25 & 0.35 & 0.45 & 0.40 & 0.45 & 0.35 & 0.35 & 0.40 & 0.45 & 0.40 & 0.40 & 0.45 & 0.60 \\
\hline 5 & 0.45 & 0.40 & 0.55 & 0.55 & 0.50 & 0.55 & 0.50 & 0.55 & 0.65 & 0.60 & 0.60 & 0.50 & 0.60 & 0.60 \\
\hline 6 & 0.60 & 0.50 & 0.70 & 0.70 & 0.95 & 0.80 & 0.70 & 0.95 & 0.65 & c. 65 & 0.60 & 0.70 & 0.85 & 0.90 \\
\hline 7 & 0.90 & 0.65 & 1.05 & 0.85 & 0.95 & 0.95 & 0,90 & 1.20 & 1.15 & 0.90 & 0.85 & 1.15 & 0.90 & 0.75 \\
\hline 8 & 0.90 & 0.75 & 1.10 & 1.20 & 1.15 & 1.05 & 1.00 & 1.35 & 1.10 & 1.05 & 1.00 & 1.25 & 1.30 & 1.00 \\
\hline 9 & 1.05 & 0.90 & $1+10$ & 1.45 & 1.20 & 1.35 & 1.15 & 1.30 & 1.40 & 1,05 & 1.50 & 1.35 & 1.25 & 1.50 \\
\hline 10 & 1.00 & 1.35 & 1.15 & 1.55 & 1.70 & 1.50 & 1.60 & 1.55 & 1.45 & 1.50 & 1.20 & 1,40 & 1.40 & 1.70 \\
\hline 11 & 1.25 & 1.40 & 1.70 & 1.50 & 1.45 & 1.45 & 1.75 & 1.65 & 1.90 & 1.60 & 1.60 & 1.50 & 1.75 & 1.70 \\
\hline 12 & 1.75 & 1.55 & 1.60 & 1.60 & 1.70 & 1.80 & 1,90 & 1.95 & 1.75 & 1.50 & 1.50 & 2.00 & 1.90 & 1.80 \\
\hline 13 & 2.00 & 1.80 & 1.75 & 1.75 & 1.65 & 1.95 & 1.85 & 2.10 & 1.95 & 1.65 & 1.75 & 2.30 & 2.00 & 1.80 \\
\hline 14 & 2.20 & 2.15 & 2.15 & 2.05 & 1.95 & 2.00 & 1.90 & 2.05 & 2.15 & 1.95 & 2,45 & 2.40 & 2.00 & 1.90 \\
\hline
\end{tabular}

Fig. 9 Exemple de stockage des mesures (massif non chargé, valeurs des $F_{z}$ )

- détermination des isobares.

Le programme informatique utilisé permet, outre le calcul des grandeurs précédentes de déterminer et donc de tracer les isobares. Pour ce faire le nombre de points de mesure est fictivement augmenté par interpolation linéaire.

\section{Exemples de résultats obtenus}

Les différents résultats que nous présentons ici sont tirés des études de Faugeras (9).

\subsection{Cas du massif non chargé}

Nous donnons figures 11 et 12 l'évolution des contraintes $\sigma_{x}$ et $\sigma_{z}$ en fonction de la profondeur pour différentes zones du massif. En ce qui concerne les contraintes $\sigma_{z}$ nous avons également reporté la courbe correspondant à $\sigma_{z}=\gamma \cdot z$ où $\gamma$ est le poids volumique apparent du matériau. II faut noter que les points expérimentaux se situent au-dessus de cette droite; ceci peut provenir d'une imprécision dans les valeurs de $\lambda$ et $\mu$ mais également d'une imprécision sur la valeur de $\gamma$ obtenue par comptage sur photographie. Les différences enregistrées proviennent surtout des contraintes résiduelles qui existent dans le massif, du fait des chargements qu'il a déjà subis.

\subsection{Cas d'un pieu encastré dans le massif}

Nous donnons ici les résultats obtenus pour un chargement sur la pointe d'un pieu encastré de $54 \mathrm{~cm}$ dans le massif.

\subsubsection{Allure des isostatiques}

En reportant en chaque point du massif où les mesures ont été effectuées la valeur de l'angle $\theta$, il est possible d'obtenir l'allure générale des isostatiques (fig. 13 et 14). En effet, dans un premier temps nous obtenons des segments de droite, mais nous pouvons ensuite en tracer l'enveloppe. Pour avoir le réseau complet, il suffit alors de tracer les orthogonales.
Les résultats obtenus semblent, aux imprécisions de mesure près, en accord avec les théories existantes. Du réseau de droites orthogonales du massif non chargé, nous aboutissons au réseau classique sous chargement.

L'exploitation des isostatiques pose encore quelques problèmes. En effet les résultats sont trop imprécis notamment au voisinage du pieu. Dans certaines zones il est quasi impossible de tracer les isostatiques, certains résultats étant contradictoires. Près de la surface du massif, les valeurs mesurées sont faibles et de ce fait l'erreur relative commise sur la détermination de $\theta$ peut être très importante. De plus, il semble que nous ne soyons pas complètement affranchis des effets de bord.

\subsubsection{Allure générale des isobares}

Sur les figures 15 et 16 , nous voyons nettement apparaître les isobares. Chaque type de caractère représente une valeur donnée de la contrainte et nous avons indiqué la correspondance au bas de chaque figure.

Nous remarquons que les "bulbes " de contrainte pour $\sigma_{z}$ ne se referment pas complètement, ce qui laisse à penser que le massif n'est pas suffisamment profond. La même observation peut être faite au niveau des isobares en $\sigma_{\mathrm{x}}$.

Il faut également noter un écrasement des isobares dû à l'interpolation linéaire qui a été nécessaire pour les tracer.

\section{Conclusion}

La méthode de mesure des contraintes au sein d'un massif analogique que nous proposons ici présente un intérêt certain. En effet c'est une méthode simple et de mise en œuvre relativement aisée.

Bien que certains points restent à améliorer notamment en ce qui concerne la diminution des dispersions, la méthode dans son état actuel peut être exploitée de manière relativement satisfaisante. Nous avons montré 


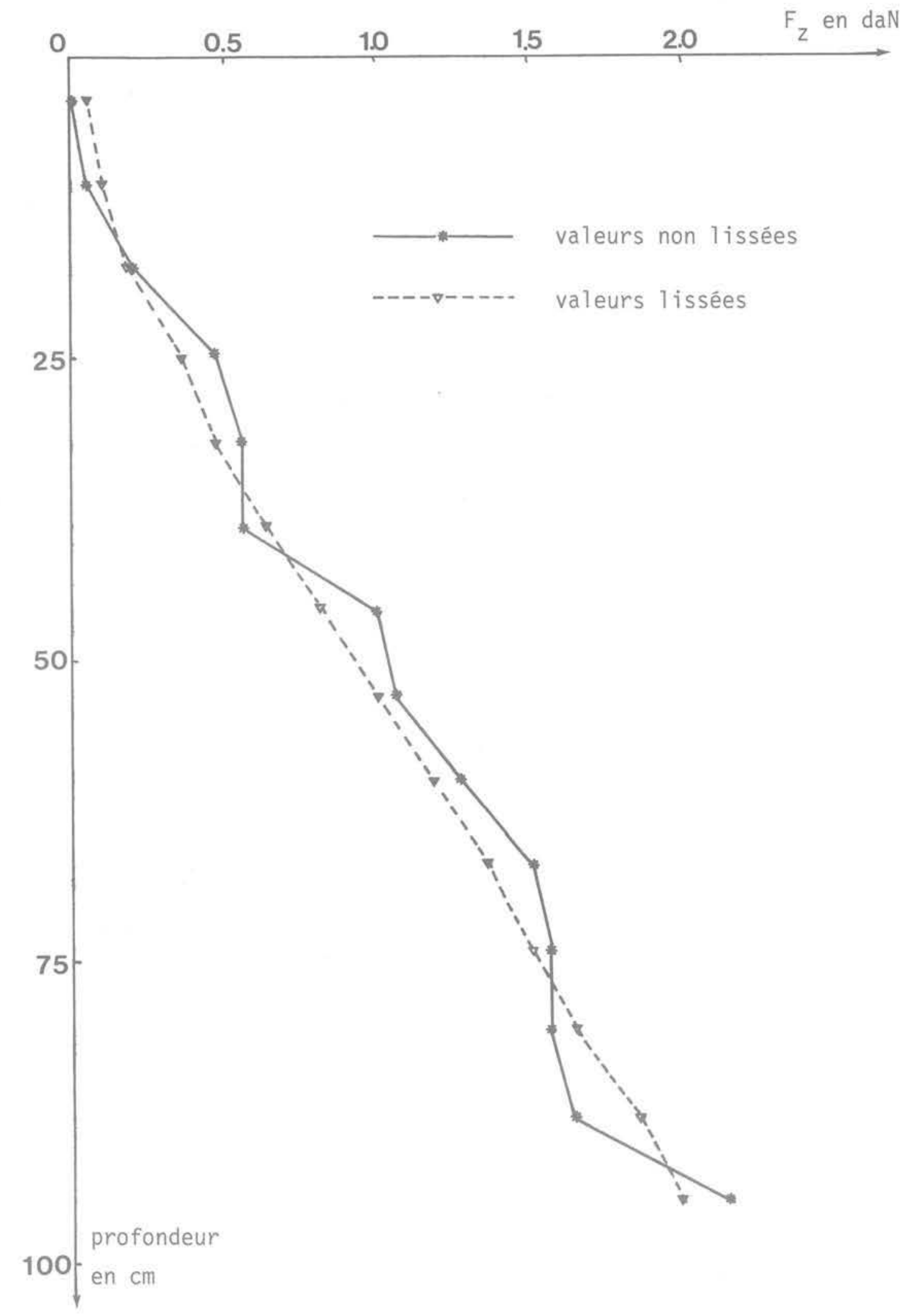

Fig. 10 Valeurs de Fz en fonction de la profondeur 


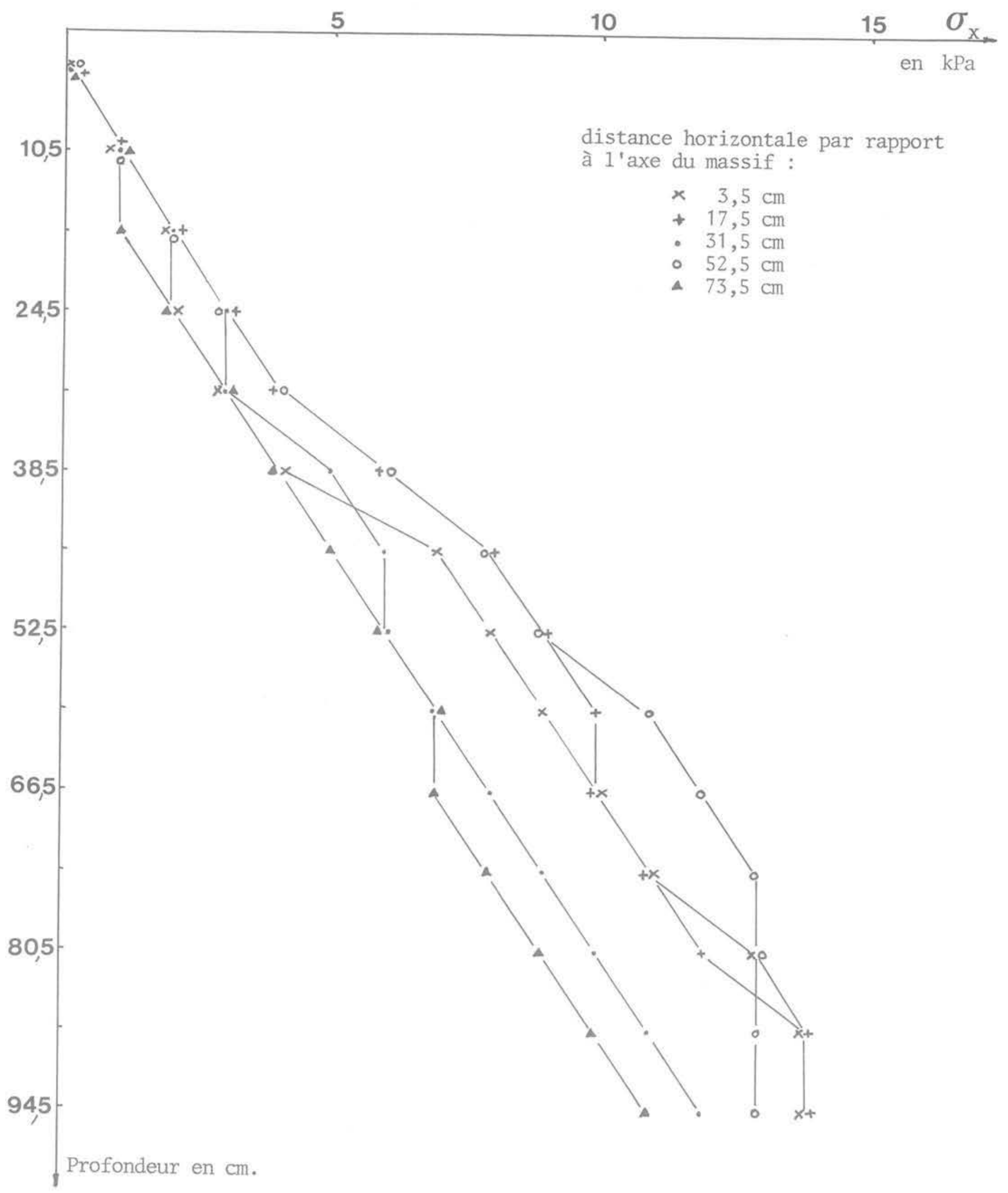

Fig. 11 Evolution des contraintes $\sigma_{x}$ en fonction de la profondeur 


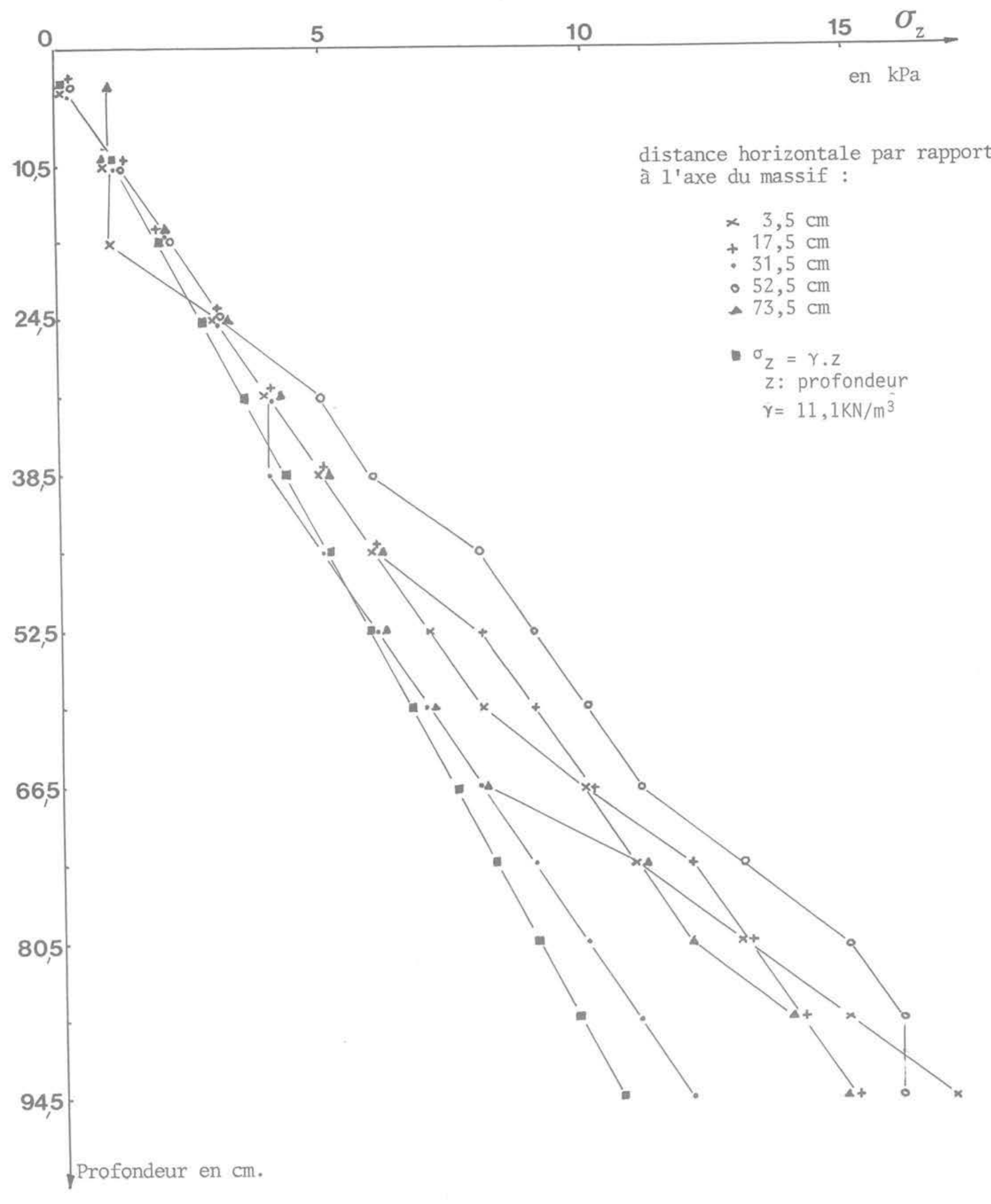

Fig. 12 Evolution des contraintes $\sigma_{z}$ en fonction de la profondeur 


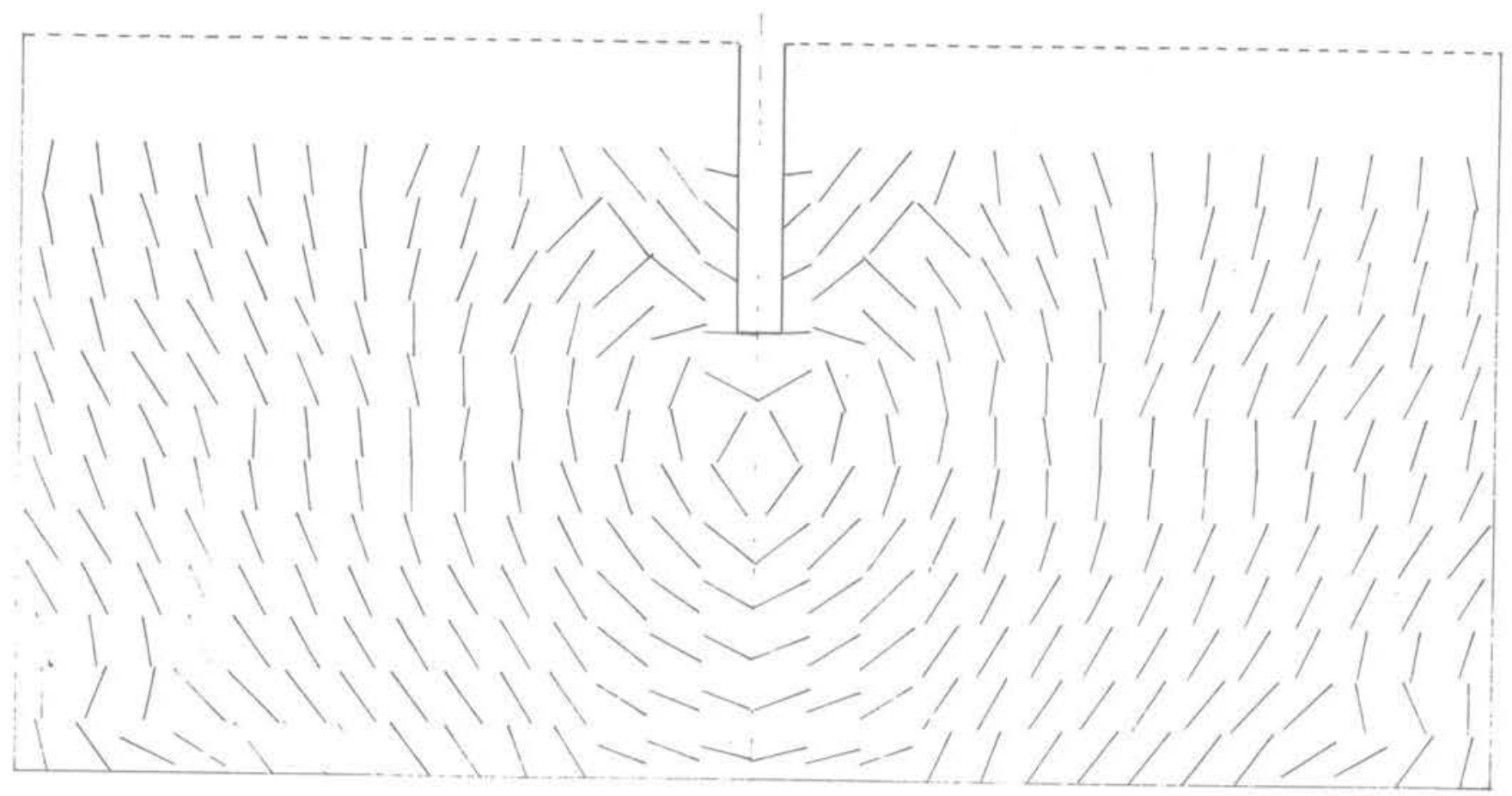

Fig. 13 Allure générale des isostatiques pour une contrainte moyenne sous la pointe du pieu de $27 \mathrm{kPa}$

Fig. 14 Isostatiques pour une contrainte moyenne sous la pointe du pieu de $90 \mathrm{kPa}$

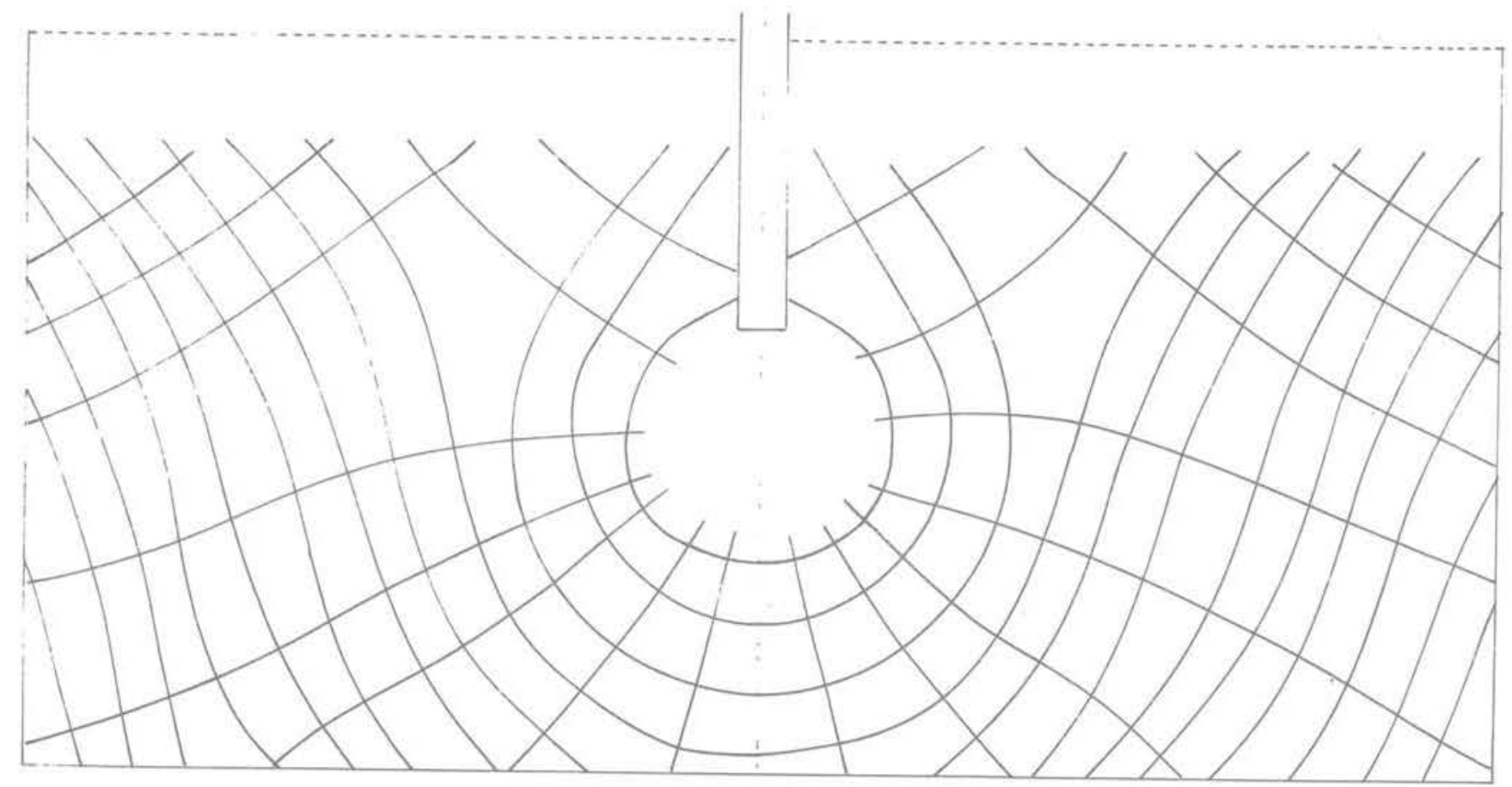

qu'elle permet d'obtenir l'état de contrainte en tous points du massif ainsi que l'allure générale des isostatiques.

L'interprétation des résultats peut conduire à la détermination des zones plastifiées et permettre l'analyse de l'évolution de ces zones au cours du chargement.

Outre la vérification de certaines théories, il est également possible de retrouver ainsi les divers chemins de contraintes suivis au cours d'essais de chargement d'ouvrages ou bien au cours de la réalisation de certains essais de mécanique des sols.

Bien que n'étant effectuée que sur des modèles bidimensionnels, nous pensons que cette méthode peut permettre d'étudier avec profit un certain nombre de problèmes de mécanique des sols dans la mesure oủ c'est, en quelque sorte, le sol qui est son propre capteur de force. Le milieu n'est donc pas modifié par la présence d'appareils de mesures susceptibles d'engendrer des effets parasites. 


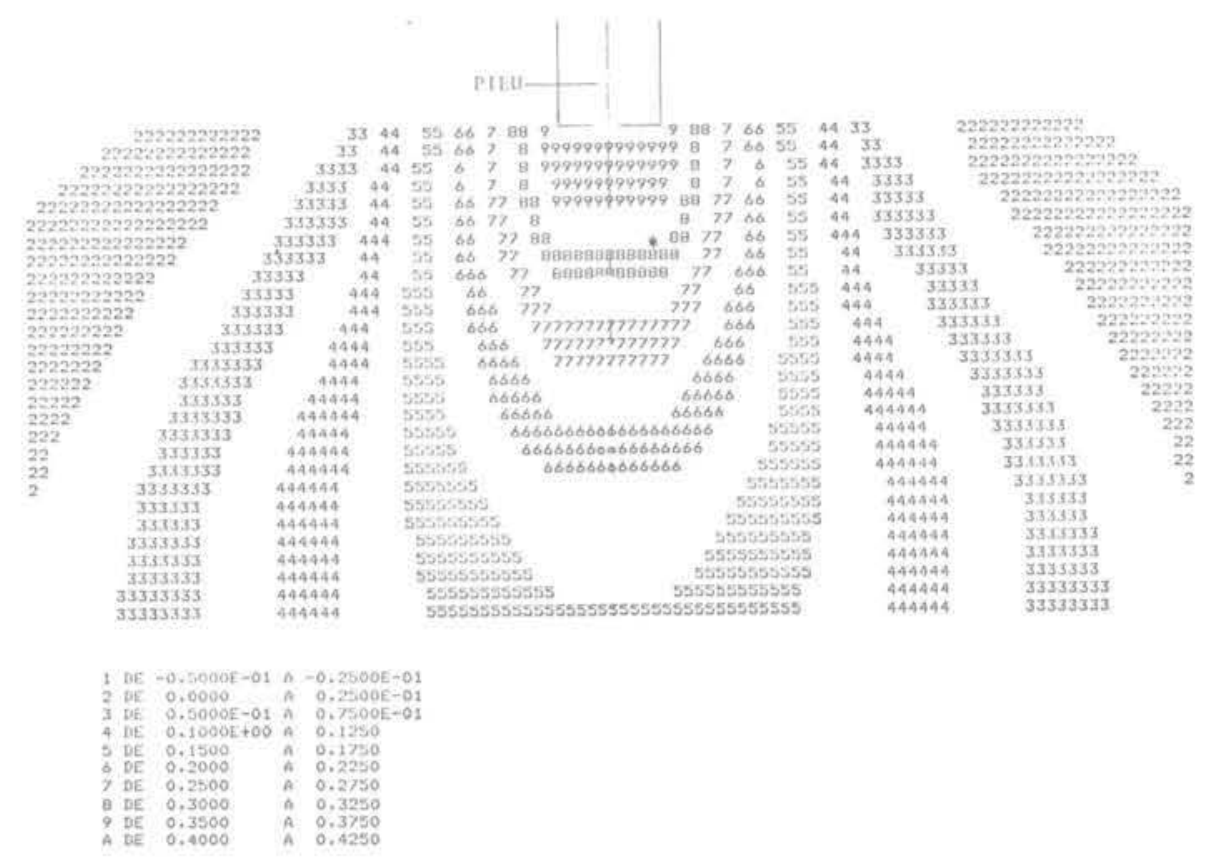

Fig. 15 Isobares en $\sigma_{z}$ pour une contrainte moyenne sous la pointe du pieu de $90 \mathrm{kPa}$

Fig. 16 Isobares en $\sigma_{X}$ pour une contrainte moyenne sous la pointe du pieu de $90 \mathrm{kPa}$

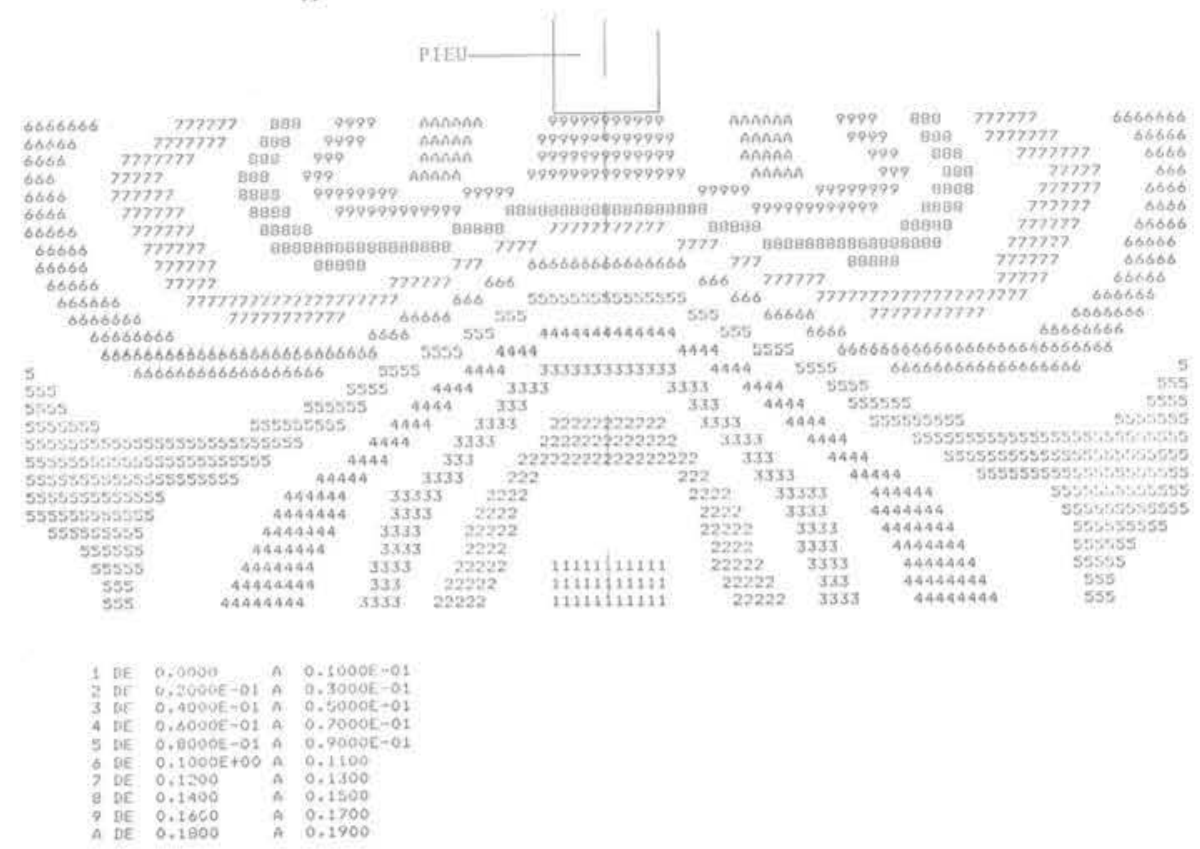

\section{Références Bibliographiques}

[1] G. Schneebeli - Une analogie mécanique pour les terres sans cohésion. Comptes rendus des séances de l'Académie des Sciences. Tome 243. Paris 1956.

[2] J. Biarez - Contribution à l'étude des propriétés mécaniques des sols et des matériaux pulvérulents. Thèse. Grenoble 1962.

[3] Nguyen Than Long, F. Schlosser, Y. Guegan, G. Legay - Étude des murs en terre armée sur modèles réduits bidimentionnels. Rapport de recherche $n^{\circ} 30$. L. C.P.C. Paris 1973.

[4] J.M. Gresillon - Étude des fondations profondes en milieu pulvérulent. Thèse. Grenoble 1970.
[5] M. Burel - Étude expérimentale de la force portante des fondations par analogie avec des rouleaux. Thèse. Grenoble 1960.

[6] W. Cichy, E. Dembicki, W. Odrobinsky, A. Tejchman, B. Zadroga - Bearing capacity of subsoil under shallow foundations study and model test. Zeszyty Naukowe Politeckniki Gdanskiej. Gdansk 1978.

[7] A. Ferri - Étude sur modèle analogique bidimensionnel du comportement d'un sol sollicité par une fondation profonde. Mémoire Cust. Clermont 1978.

[8] C. Behnia - Étude des voûtes en terre armée. Thèse. Paris VI 1972.

[9] J.C. Faugeras - L'essai de compressibilité des sols au pénétromètre statique et son interprétation sur modèle analogique. Thèse. Toulouse 1979. 